\title{
Belief Change and 3-Valued Logics: Characterization of 19,683 Belief Change Operators
}

\author{
Nerio Borges \\ Escuela Politécnica Nacional, Departamento de Matemáticas \\ Av. Ladrón de Guevara 253, Quito 170517, Ecuador \\ Ramón Pino Pérez \\ Universidad de Los Andes, Departamento de Matemáticas \\ Facultad de Ciencias, Mérida 5101, Venezuela.
}

PINO@ULA.VE

\begin{abstract}
In this work we introduce a 3-valued logic with modalities, with the aim of having a clear and precise representation of epistemic states, thus the formulas of this logic will be our epistemic states. Indeed, these formulas are identified with ranking functions of 3 values, a generalization of total preorders of three levels. In this framework we analyze some types of changes of these epistemic structures and give syntactical characterizations of them in the introduced logic. In particular, we introduce and study carefully a new operator called Cautious Improvement operator. We also characterize all operators that are definable in this framework.
\end{abstract}

\section{Introduction}

Classical propositional logic is the most common choice when studying and modelling belief change operators (see for instance Gärdenfors, 1992; Peppas, 2008; Pino Pérez \& Uzcátegui, 2010). In the classical AGM framework (Alchourrón et al., 1985; Gärdenfors, 1988), for instance, epistemic states are represented by theories and new pieces of information by propositional formulas. In the Katsuno and Mendelzon framework (1991) (denoted KM hereafter), on the other hand, epistemic states as well as new pieces of information are propositional formulas. In both AGM and KM settings, the new information is intended to express a fact about the world and this new knowledge must always be included in the epistemic state resulting from the revision process.

A very useful tool for understanding the logical model of revision operators is Katsuno and Mendelzon's Representation Theorem (Theorem 3.3, 1991). It says that revision operators are represented by assignments mapping epistemic states to total preorders over interpretations and the output is a formula or theory having as models the minimal models of the new piece of information with respect to the preorder associated to the old epistemic state. As a matter of fact, this tool is exploited by Darwiche and Pearl (1997), to shift the notion of epistemic state to a more abstract one, where the paradigm of epistemic state is indeed that of a total preorder over interpretations. In our view, this work together with Boutilier's Natural Revision (1996) is one of the most influential in a series of works trying to capture some types of semantical behavior via a syntactical characterization (Booth \& Meyer, 2006; Booth et al., 2006; Jin \& Thielscher, 2007; Konieczny et al., 2010; Konieczny \& Pino Pérez, 2008; Medina Grespan \& Pino Pérez, 2013; Nayak, 1994; Rott, 2009). In most 
of these works the notion of epistemic state is quite general and classical logic is used to express properties of the beliefs (the logical visible part of an epistemic state). However, the most representative structures of epistemic states are total preorders over interpretations.

In this work, we follow this tradition but in a new way. Our aim is, first of all, to have a complete logic representation of epistemic states and then to be able to describe change operators in this logic. Thus, we want the semantical structures of preorders to be represented in a logical language. In order to do that, we introduce a 3-valued logic with modalities where the formulas can be identified with a generalisation of total preorders of three levels: a ranking function mapping interpretations into truth values.

The typical situations we want to model in our framework are illustrated in the following example.

Example 1. Sébastien is reasoning dynamically about the following fact: the chloroquine cures the coronavirus. At first, he rejects completely this fact. Now he reads in a newspaper that a reputed French Doctor claims that the fact is true. After that, given that he is quite skeptical, he is dubious about the fact. He doesn't accept nor reject the fact. He has a cautious attitude. At this moment, he is uncertain about the fact. After that, he read that Japanese Doctors have been using the chloroquine to treat the coronavirus successfully. Now, he ends up accepting this fact.

In his lockdown Sébastien has more time for reading the news in the web, and he reads that the experiments until now are not concluding for acceptation or rejection of the fact. After that, Sébastien is uncertain about the fact.

Note that if Sébastien is accepting the fact and he is informed that the fact is false, he doesn't reject the fact and, by a sort of inertia, he thinks that the fact is uncertain.

Thus, in order to model situations like in our previous example, we suppose that the agents are working within a finite propositional 3 -valued logic on $n$ variables. One value represents acceptation, the second value represents rejection and the third value represents uncertainty. Each variable in this logic, as customary, represents an atomic "fact" about the world. We chose a 3-valued logic because this agent does not necessarily have an opinion -or knowledge- about every single atomic fact.

In a general manner, we suppose that our agents classify all the possible worlds, i.e., all the truth assignments into three blocks $L_{1}, L_{2}$ and $L_{3}$. Those in $L_{1}$ are the most plausible scenarios. Assignments in $L_{2}$ are assignments about which the agent is uncertain. She doesn't know whether to accept or reject these worlds. At the level $L_{3}$ the agent puts the worlds which she considers unlikely. Note that naturally this induces a total preorder over interpretations: we order the interpretations following the level of acceptance, that is interpretations in $L_{1}$ are preferred to interpretations in $L_{2}$ or $L_{3}$, and interpretations in $L_{2}$ are preferred to interpretations in $L_{3}$. These structures will be the epistemic states of the agents.

In the same manner as propositional classical logic captures all the structures at two levels (accepted or rejected) ${ }^{1}$, we present here a logic in which the formulas capture all the three levels' structures. In order to do that, we propose here the use of the Kleene Strong

1. Note that two levels' structures (ranking functions of two values) are more general than total preorders having at most two levels. This is because we can have empty levels. For instance, if the first level is empty, this corresponds to a contradiction; if the second level is empty, this corresponds to a tautology. 
three valued logic (Bergmann, 2008) plus two modalities which will be necessary for the completeness of the representation.

Thus, with the help of this logic, we can model belief change under uncertainty. In order to understand the mechanisms which govern the changes, we begin with a particular operator we call Cautious Improvement which captures the features of Example 1. The way to describe the changes produced in the old epistemic state by the new piece of information is reminiscent of the changes produced by the improvement operators introduced by Konieczny and Pino Pérez (2008) (see also Konieczny et al., 2010; Medina Grespan \& Pino Pérez, 2013).

It is worth to note that in our framework, both the (old) epistemic state and the new piece of information are formulas. Since they are formulas in our new logic, they are both (complex) epistemic states. Therefore, we return, in a natural way, to the paradigm of revising an epistemic state by an epistemic state first proposed by Benferhat et al. (2000).

The Cautious Improvement operator is characterized syntactically. An analysis of the techniques involved in the definition and in the syntactical characterization allows us to find a general method for defining all the operators under uncertainty and extract syntactic postulates that characterize them.

This work is organized as follows. In Section 2 the Kleene strong 3-valued logic with two new modalities is defined. Therein it is established that every ranking function mapping interpretations into an ordered scale of three elements can be represented by a formula of this new logic. Section 3 is devoted to the definition of the Cautious Improvement operator and its syntactic characterization. In Section 4 we characterize each of the $3^{9}(19,683)$ possible change operators in our logic. In Section 5 we compare our results with other works in the literature. In Section 6, we conclude with some remarks and give some lines for future development of this work. Finally, in Appendix A we give all the proofs, namely a combinatorial proof of the necessity of our two modalities in order to be able to represent all ranking functions over the interpretations into three values (Theorem 2).

\section{The Logic}

We work within a modal variant of finite $K_{3}^{S}$, the Kleene strong 3-valued logic (Kleene, 1938; see also Bergmann, 2008), with variables $x_{0}, x_{1}, \ldots, x_{n-1}$. We will call $\operatorname{Var}_{n}$ the set of these variables. The usual version of $K_{3}^{S}$ has the same syntax as classical propositional logic. For the semantics, we have three possible truth values: 1 representing truth, 0 for falsehood and 1/2 for non-determined. The truth tables for the connectives are the following:

These two structures are identically represented as a flat preorder. Thus, total preorders are less rich structures than two levels structures. Idem for three levels structures. 


\begin{tabular}{c|c|c}
$P$ & $Q$ & $P \wedge Q$ \\
\hline 1 & 1 & 1 \\
1 & $1 / 2$ & $1 / 2$ \\
1 & 0 & 0 \\
$1 / 2$ & 1 & $1 / 2$ \\
$1 / 2$ & $1 / 2$ & $1 / 2$ \\
$1 / 2$ & 0 & 0 \\
0 & 1 & 0 \\
0 & $1 / 2$ & 0 \\
0 & 0 & 0
\end{tabular}

\begin{tabular}{c|c|c}
$P$ & $Q$ & $P \vee Q$ \\
\hline 1 & 1 & 1 \\
1 & $1 / 2$ & 1 \\
1 & 0 & 1 \\
$1 / 2$ & 1 & 1 \\
$1 / 2$ & $1 / 2$ & $1 / 2$ \\
$1 / 2$ & 0 & $1 / 2$ \\
0 & 1 & 1 \\
0 & $1 / 2$ & $1 / 2$ \\
0 & 0 & 0 \\
& & \\
& $P$ & $\neg P$ \\
\cline { 2 - 3 } & 1 & 0 \\
& $1 / 2$ & $1 / 2$ \\
& 0 & 1
\end{tabular}

\begin{tabular}{c|c|c}
$P$ & $Q$ & $P \longrightarrow Q$ \\
\hline 1 & 1 & 1 \\
1 & $1 / 2$ & $1 / 2$ \\
1 & 0 & 0 \\
$1 / 2$ & 1 & 1 \\
$1 / 2$ & $1 / 2$ & $1 / 2$ \\
$1 / 2$ & 0 & $1 / 2$ \\
0 & 1 & 1 \\
0 & $1 / 2$ & 1 \\
0 & 0 & 1
\end{tabular}

As usual, given an interpretation $\omega$ and a formula $\varphi$ of $K_{3}^{S}$, we denote $\omega(\varphi)$ the value of the formula $\varphi$ in the interpretation $\omega$, calculated recursively using the truth tables.

For the sake of brevity, let's say that a valuation $\omega$ is a quasi-model of $\theta$ iff $\omega(\theta)=1 / 2$ and a countermodel of $\theta^{\prime}$ if $\omega\left(\theta^{\prime}\right)=0$. Then we say that $\omega$ is a quasi-model of a set $\Sigma$ iff it is a quasi-model for all the formulas in $\Sigma$. The notion of model is the same as in classic propositional logic. That is, $\omega$ is a model of $\theta$ iff $\omega(\theta)=1$. A formula is a contradiction if it only has countermodels.

Regarding semantics, we have to define some usual symbols:

1. For two formulas $\alpha, \beta$ we write $\equiv$ when they have the same truth table.

2. We write $\alpha \vDash \beta$ when every model of $\alpha$ is a model of $\beta$, i.e., we use this symbol with its classic interpretation.

3. We write $\alpha \vDash \beta$ to abbreviate $\alpha \vDash \beta$ and $\beta \vDash \alpha$.

Note that the meaning of the symbols $=$ 作 and $\equiv$ is quite different. Indeed, when $\alpha \equiv \beta$, their sets of models, quasi-models and countermodels are exactly the same. Whereas $\alpha=\beta$ means only that the set of models of both formulas coincide.

An interesting aspect of this logic is that there are no tautologies. Actually, it is easy to check that for any interpretation $\omega$ taking the constant value $\frac{1}{2}$, we have that for every formula $\varphi, \omega(\varphi)=\frac{1}{2}$.

We extend $K_{3}^{S}$ by adding the modal operators $\diamond_{1}$ and $\square_{1}$. We include also the symbols ' $\perp$ ' and ' $T$ ' as the logical constants that always evaluate to 0 and to 1 , respectively. Our syntax is also extended by the new formation rule stating that if $\varphi$ is a formula then $\nabla_{1} \varphi$ and $\square_{1} \varphi$ are also formulas. The modal operators are interpreted as follows:

\begin{tabular}{c|c}
$\varphi$ & $\nabla_{1} \varphi$ \\
\hline 1 & $1 / 2$ \\
$1 / 2$ & 0 \\
0 & 0
\end{tabular}

\begin{tabular}{c|c}
$\varphi$ & $\square_{1} \varphi$ \\
\hline 1 & 1 \\
$1 / 2$ & 1 \\
0 & $1 / 2$
\end{tabular}


Intuitively, $\diamond_{1}$ worsens the truth value of $\varphi$ and $\square_{1}$ improves it. It is easily noted that $\square_{1} \varphi=\neg \nabla_{1} \neg \varphi$, so these are dual operators.

Note that in $K_{3}^{S}$ extended by $\square_{1}$ there are many tautologies. As a matter of fact, for every formula $\varphi$, the formula $\square_{1} \square_{1} \varphi$ is a tautology.

With the use of $\nabla_{1}$ and $\square_{1}$ we can find, given a truth assignment $\omega$, a formula $\varphi_{\omega}$ such that its only model is $\omega$. Indeed, given a truth assignment $\omega=\left\langle t_{0}, t_{1}, \ldots, t_{n-1}\right\rangle$ on Var, we have the formula

$$
\varphi_{\omega}:=\alpha_{0} \wedge \alpha_{1} \wedge \cdots \wedge \alpha_{n-1}
$$

where each $\alpha_{i}$ is a formula given by

$$
\alpha_{i}=\left\{\begin{array}{lll}
x_{i} & \text { if } \quad t_{i}=1 \\
\neg x_{i} & \text { if } \quad t_{i}=0 \\
\square_{1} x_{i} \wedge \square_{1} \neg x_{i} & \text { if } \quad t_{i}=1 / 2
\end{array}\right.
$$

This formula evaluates to 1 if, and only if, all of the $\alpha_{i}$ 's evaluate to 1 , thus the only model for $\varphi_{\omega}$ is $\omega$. Notice that it can have more than one quasi-model. On the other hand, if $\left\{\omega_{0}, \omega_{1}, \ldots, \omega_{k-1}\right\}$ is a set of truth assignments, the formula

$$
\varphi_{\omega_{0}, \omega_{1}, \ldots, \omega_{n-1}}:=\bigvee_{0 \leq i \leq n-1} \varphi_{\omega_{i}}
$$

has the interpretations in $\left\{\omega_{0}, \omega_{1}, \ldots, \omega_{k-1}\right\}$ as its only models.

We can also give "normal" forms that allow us to "push" the modal operator $\diamond_{1}$ within parentheses. One can easily check that

$$
\diamond_{1}\left(\theta_{1} \wedge \theta_{2}\right) \equiv \diamond_{1} \theta_{1} \wedge \diamond_{1} \theta_{2}
$$

and that

$$
\diamond_{1}\left(\theta_{1} \vee \theta_{2}\right) \equiv \diamond_{1} \theta_{1} \vee \diamond_{1} \theta_{2}
$$

On the other hand, for reasons that will become apparent later, we are going to introduce the modal operator $\diamond_{2}$ and its dual $\square_{2}$ whose semantics are given by the truth tables

\begin{tabular}{c|c}
$\varphi$ & $\diamond_{2} \varphi$ \\
\hline 1 & 1 \\
$1 / 2$ & 0 \\
0 & 0
\end{tabular}

\begin{tabular}{c|c}
$\varphi$ & $\square_{2} \varphi$ \\
\hline 1 & 1 \\
$1 / 2$ & 1 \\
0 & 0
\end{tabular}

It is also easy to check that

$$
\diamond_{2}\left(\theta_{1} \wedge \theta_{2}\right) \equiv \diamond_{2} \theta_{1} \wedge \diamond_{2} \theta_{2}
$$

and

$$
\diamond_{2}\left(\theta_{1} \vee \theta_{2}\right) \equiv \diamond_{2} \theta_{1} \vee \diamond_{2} \theta_{2}
$$

We denote by $K_{3}^{S}+\diamond_{1}+\diamond_{2}$ the modal extension of $K_{3}^{S}$ by $\diamond_{1}$ and $\diamond_{2}$ and by $K_{3}^{S}+\diamond_{i}$ the extension by $\nabla_{i}$ for $i=1,2$. The set of all the formulas in this logic is denoted by $\mathcal{F}$.

Again, given an interpretation $\omega$ and a formula $K_{3}^{S}+\diamond_{1}+\diamond_{2}$, we denote as $\omega(\varphi)$ the value of the formula $\varphi$ in the interpretation $\omega$, calculated, as usual, recursively using the truth tables. 


\subsection{Formulas, Ranking Functions and Preorders}

In the finite case, the logic $K_{3}^{S}+\diamond_{1}+\diamond_{2}$ over the set of variables

$$
\operatorname{Var}_{n}=\left\{x_{0}, x_{1}, \ldots, x_{n-1}\right\}
$$

the set of all interpretations is

$$
\mathcal{I}_{n}=\left\{\omega_{0}, \omega_{1}, \ldots, \omega_{3^{n}-1}\right\}
$$

Each formula $\varphi$ in $K_{3}^{S}+\diamond_{1}+\diamond_{2}$ induces a partition of $\mathcal{I}_{n}$ into three blocks $L_{1}(\varphi), L_{2}(\varphi)$ and $L_{3}(\varphi)$ defined as

$$
\begin{aligned}
& L_{1}(\varphi)=\left\{\omega \in \mathcal{I}_{n}: \omega(\varphi)=1\right\} \\
& L_{2}(\varphi)=\left\{\omega \in \mathcal{I}_{n}: \omega(\varphi)=1 / 2\right\} \\
& L_{3}(\varphi)=\left\{\omega \in \mathcal{I}_{n}: \omega(\varphi)=0\right\}
\end{aligned}
$$

We call these blocks levels, and we call $L_{j}(\varphi)$ the $j$-th level. Thus, the first level contains the worlds accepted by $\varphi$; the second level contains the uncertain worlds of $\varphi$ and the third level contains the worlds rejected by $\varphi$.

Note that this partition can be seen as a ranking function $r_{\varphi}: \mathcal{I}_{n} \longrightarrow\left\{0, \frac{1}{2}, 1\right\}$, where $r_{\varphi}(\omega)=1$ when $\omega \in L_{1}(\varphi), r_{\varphi}(\omega)=\frac{1}{2}$ when $\omega \in L_{2}(\varphi)$ and $r_{\varphi}(\omega)=0$ when $\omega \in L_{3}(\varphi)$.

As a matter of fact, in a general manner, it is easy to see that there is a one to one correspondence between the partitions into three levels and the ranking functions taking three values. Actually, given a ranking function $r: \mathcal{I}_{n} \longrightarrow\left\{0, \frac{1}{2}, 1\right\}$ we use the notation $L_{j}(r)$ for the levels of the partition associated to it, that is, $L_{1}(r)=r^{-1}(1), L_{2}(r)=r^{-1}\left(\frac{1}{2}\right)$ and $L_{3}(r)=r^{-1}(0)$.

Note also that we can associate, in a natural way, a binary relation $\preceq_{r}$ on $\mathcal{I}_{n}$ to a ranking function $r: \mathcal{I}_{n} \longrightarrow\left\{0, \frac{1}{2}, 1\right\}$ as follows:

$$
\omega \preceq_{r} \omega^{\prime} \Longleftrightarrow r(\omega) \geq r\left(\omega^{\prime}\right) \Longleftrightarrow \omega \in L_{i}(r), \omega^{\prime} \in L_{j}(r) \quad \text { with } \quad i \geq j
$$

The reader can easily check that it is a total preorder, i.e., a binary relation which is reflexive, transitive and total.

In this way, we can associate a total preorder to a formula $\varphi$ of $K_{3}^{S}+\diamond_{1}+\diamond_{2}$, namely, the total preorder $\preceq_{r_{\varphi}}$, which will be denoted $\preceq_{\varphi}$ in order to simplify the notation.

Here it is important to note that equivalent formulas have exactly the same ranking function. Moreover, two formulas are equivalent if and only if their ranking functions coincide, that is,

$$
\varphi \equiv \theta \Longleftrightarrow r_{\varphi}=r_{\theta}
$$

Therefore, the ranking functions associated to formulas characterize the logical equivalence. However, this is not the case with the associated preorders. Of course, if two formulas are equivalent their associated preorders coincide. But the converse is not true. Indeed, we can have formulas far from being equivalent, like $\perp$ and $T$, having the same associated preorder: the flat preorder. Thus, the semantic representation of formulas as ranking functions is richer and, by far, more precise than the preorder representation. 
As a matter of fact, our logic is complete, concerning the ranking functions. We will prove that for every ranking function $r: \mathcal{I}_{n} \longrightarrow\left\{0, \frac{1}{2}, 1\right\}$ there is a formula in $K_{3}^{S}+\diamond_{1}+\diamond_{2}$ having $r$ as its associated ranking function.

By abuse of notation, in this work we will continue to call three-level preorder, any partition in three levels given by a ranking function $r: \mathcal{I}_{n} \longrightarrow\left\{0, \frac{1}{2}, 1\right\}$. Thus, ranking function, three-level preorder and partition in three levels are synonyms in this work.

Theorem 1. Given a ranking function $r: \mathcal{I}_{n} \longrightarrow\left\{0, \frac{1}{2}, 1\right\}$, there is a formula $\varphi_{r}$ in $K_{3}^{S}+$ $\diamond_{1}+\diamond_{2}$ such that $r=r_{\varphi_{r}}$.

Theorem 1 shows that every three level preorder on $I_{n}$ is characterized by a formula in $K_{3}^{S}+\diamond_{1}+\diamond_{2}$.

In Theorem 2 we establish that both $\diamond_{1}$ and $\diamond_{2}$ are needed for this.

Theorem 2. $K_{3}^{S}+\diamond_{i}$ with $i=1,2$ is not enough to define every 3-level preorder on $\mathcal{I}_{n}$.

As a straightforward consequence of the previous theorem, we can see that the logic $K_{3}^{S}$ is not rich enough to represent all ranking functions into three values.

Corollary 1. There are three-level preorders on all the interpretations on $\mathbf{V a r}_{n}$ that cannot be defined by any formula in $K_{3}^{S}$.

This is an important difference between $K_{3}^{S}$ and classical logic which is enough to represent all the two levels preorders.

\section{The Cautious Improvement Operator}

We want to define a belief revision operator of the kind

$$
*: \mathcal{F} \times \mathcal{F} \longrightarrow \mathcal{F}
$$

Thus, in this framework we are going to represent both epistemic states and epistemic inputs by formulas in $K_{3}^{S}+\diamond_{1}+\diamond_{2}$. Also the outputs will be represented by the same type of formulas. As usual, $*(\varphi, \theta)$, is written as $\varphi * \theta$.

\subsection{Definition of the Operator}

We exploit the representation of formulas as ranking functions in order to define our operators. Thus, for a fixed $n \in \mathbb{N}$ we will define $*$ as a binary operator on ranking functions mapping $\mathcal{I}_{n}$ into $\left\{1, \frac{1}{2}, 0\right\}$.

Definition 1. Let $*: \mathcal{F} \times \mathcal{F} \longrightarrow \mathcal{F}$ an operator. This operator is said to be the Cautious Improvement operator if and only if $r_{\varphi}, r_{\theta}$ and $r_{\varphi * \theta}$ satisfy the following table:

\begin{tabular}{c|c|c|c}
$\omega$ & $L_{1}\left(r_{\theta}\right)$ & $L_{2}\left(r_{\theta}\right)$ & $L_{3}\left(r_{\theta}\right)$ \\
\hline$L_{1}\left(r_{\varphi}\right)$ & $L_{1}\left(r_{\varphi * \theta}\right)$ & $L_{2}\left(r_{\varphi * \theta}\right)$ & $L_{2}\left(r_{\varphi * \theta}\right)$ \\
\hline$L_{2}\left(r_{\varphi}\right)$ & $L_{1}\left(r_{\varphi * \theta}\right)$ & $L_{2}\left(r_{\varphi * \theta}\right)$ & $L_{3}\left(r_{\varphi * \theta}\right)$ \\
\hline$L_{3}\left(r_{\varphi}\right)$ & $L_{2}\left(r_{\varphi * \theta}\right)$ & $L_{2}\left(r_{\varphi * \theta}\right)$ & $L_{3}\left(r_{\varphi * \theta}\right)$
\end{tabular}

The previous table completely describes the level of every interpretation $\omega$ in $r_{\varphi * \theta}$ given the levels where it is located in $r_{\varphi}$ and $r_{\theta}$. 
We have defined $*$ in a semantical way. Thanks to Theorem 1 , we know that there is a formula in $K_{3}^{S}+\diamond_{1}+\diamond_{2}$ having the ranking function $r_{\varphi * \theta}$ described by Table 2 . Thus, $*$ is well defined up to logical equivalence.

It is important to note that Table 2 yields the following truth table for $\varphi * \theta$ :

\begin{tabular}{c|c|c}
$\varphi$ & $\theta$ & $\varphi * \theta$ \\
\hline \hline 1 & 1 & 1 \\
\hline 1 & $1 / 2$ & $1 / 2$ \\
\hline 1 & 0 & $1 / 2$ \\
\hline $1 / 2$ & 1 & 1 \\
\hline $1 / 2$ & $1 / 2$ & $1 / 2$ \\
\hline $1 / 2$ & 0 & 0 \\
\hline 0 & 1 & $1 / 2$ \\
\hline 0 & $1 / 2$ & $1 / 2$ \\
\hline 0 & 0 & 0
\end{tabular}

Figure 1 illustrates graphically the behavior of this operator.

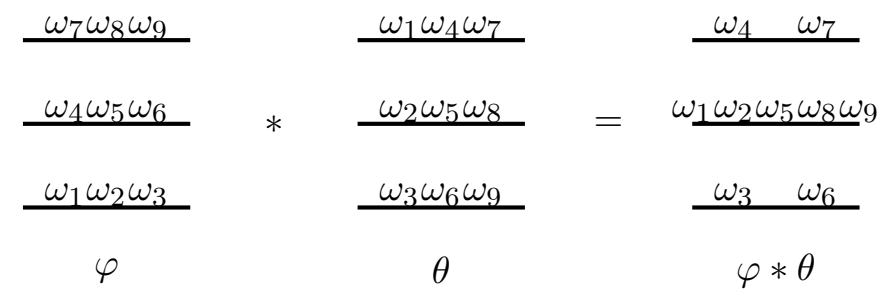

Figure 1: Cautious Improvement operator's behavior captured graphically.

\subsection{Postulates and Characterization}

In this section we will establish some postulates in terms of formulas of the logic $K_{3}^{S}+\diamond_{1}+\diamond_{2}$ which characterizes the Cautious Improvement operator.

Observation 1. Note that for every formula $\varphi$ of $K_{3}^{S}+\diamond_{1}+\diamond_{2}$, the formula $\square_{1} \varphi$ captures the first two levels of $\varphi$. More precisely, for every interpretation $\omega$, we have that $\omega$ is a model of $\square_{1} \varphi$ if and only if $\omega$ is a model of $\varphi$ or $\omega(\varphi)=\frac{1}{2}$.

Now, we consider the following postulate:

$$
\varphi * \theta=\equiv \square_{1} \varphi \wedge \theta
$$

By Observation 1, this postulate says that the models of the revision of $\varphi$ by $\theta$ are exactly the models of $\theta$ which are in some of the two first levels of $\varphi$, that is, the models of the new information which are not rejected by the old information.

Observation 2. Note that the models of $\neg \varphi$ are exactly the interpretations rejected by $\varphi$ (the countermodels). 
We also consider the following postulate:

$$
\neg(\varphi * \theta)=\vDash \square_{1} \neg \varphi \wedge \neg \theta
$$

By Observation 2 and Observation 1, this postulate says that the countermodels of the revision of $\varphi$ by $\theta$ are exactly the countermodels of $\theta$ which are in some of the two first levels of $\neg \varphi$, that is, the countermodels of the new information which are not accepted by the old information.

Observation 3. Note that the interpretations which are uncertain for $\varphi$ are exactly the models of $\square_{1} \varphi \wedge \square_{1} \neg \varphi$.

With the help of the previous observation and following Table 2, we can make a literal interpretation of the models of $\varphi * \theta$ which leads to the following postulate:

$$
\varphi * \theta=\vDash(\varphi \wedge \theta) \vee\left(\left(\square_{1} \varphi \wedge \square_{1} \neg \varphi\right) \wedge \theta\right)
$$

This postulate says that the models of the revision of $\varphi$ by $\theta$ are either the models of conjunction of both formulas or the models of the new formula which are uncertain for the old information.

In an analogous manner, using Table 2 , we can consider literally the rejected models of $\varphi * \theta$. This leads to the following postulate:

$$
\neg(\varphi * \theta)=\equiv\left(\left(\square_{1} \varphi \wedge \square_{1} \neg \varphi\right) \wedge \neg \theta\right) \vee(\neg \varphi \wedge \neg \theta)
$$

This postulates says that the countermodels of the revision of $\varphi$ by $\theta$ are either the interpretations rejected by both formulas or the interpretations rejected by the new formula which are uncertain for the old information.

As a matter of fact, we have the following result:

Theorem 3. Postulates CI1 and CI2 are respectively equivalent to CI1' and CI2'.

Moreover, we have the following characterization:

Theorem 4. Postulates CI1 and CI2 characterize the Cautious Improvement operator. That is, the Cautious Improvement operator satisfies CI1 and CI2 and, conversely, if an operator satisfies CI1 and CI2, then this operator is precisely the Cautious Improvement operator.

\subsection{Other Postulates}

We will now consider other postulates. Some of them are new, others are related to postulates proposed in the belief change literature.

First we consider the coherence principle (or non contradiction principle). If the new information is coherent, that is, it is not contradictory, then the result of applying the operator with this new information is also coherent. More precisely:

$$
\text { If } \theta \text { is not a contradiction, then } \varphi * \theta \text { is not a contradiction }
$$

This principle is one of the rationality properties that good belief change operators have to satisfy. It is easy to check using the truth table, that the Cautious Improvement operator satisfies this principle. 
Another well known principle is the success principle considered for revision operators. The new information has to prevail after revision. More formally:

$$
\varphi * \theta \vDash \theta
$$

Again, it is easy to check, using the truth table of Cautious Improvement operator, that the success postulate is satisfied by this operator.

Now we are considering one principle concerning the iteration by the same information. It is a strong form of the principle of iteration success of improvement operators (Konieczny et al., 2010; Konieczny \& Pino Pérez, 2008; Medina Grespan \& Pino Pérez, 2013).

$$
(\varphi * \theta) * \theta \equiv \theta
$$

This principle says that two iterations by the same epistemic state are enough to attain exactly this epistemic state. Using the truth table, it is easy to check that the Cautious Improvement operator satisfies this postulate.

Another very natural rational principle is the idempotency principle: an information revised by itself remains unchanged.

$$
\theta * \theta \equiv \theta
$$

The Cautious Improvement operator satisfies this postulate. This can be seen through the truth table of this operator.

An interesting postulate concerning the negation is the following one: the negation of a revision is the revision of negations. More formally:

$$
\neg(\varphi * \theta) \equiv \neg \varphi * \neg \theta
$$

This postulate evokes a de Morgan's law in which the dual of the operator is the operator itself. This postulate is new in the literature. Our Cautious Improvement operator satisfies this postulate as can be easily checked with the truths tables.

Finally we are going to consider a postulate, concerning the trace of the old information in the result of revision. Actually the postulate says how to recover a part of the old information after revision. More precisely:

$$
\varphi \vDash \square_{1}(\varphi * \theta)
$$

This postulate, which is also new in the literature, is satisfied by the Cautious Improvement operator. This can be seen through the truth tables. Thus, putting together our previous discussion, we have the following result:

Theorem 5. The Cautious Improvement operator satisfies postulates CI3-CI8. 


\section{General Definition of a Belief Change Operator}

Now we want to define general belief change operators of the kind

$$
\otimes: \mathcal{F} \times \mathcal{F} \longrightarrow \mathcal{F}
$$

In order to do this, we follow the idea used for defining the Cautious Improvement operator (Definition 1). Now, we generalize Table 2 to a more abstract framework.

Definition 2. Given a function $k:\{1,2,3\}^{2} \longrightarrow\{1,2,3\}$ we define the binary operator $\otimes_{k}: \mathcal{F} \times \mathcal{F} \longrightarrow \mathcal{F}$ in the following way: given the ranking functions $r_{\varphi}, r_{\theta}$, the ranking function $r_{\varphi \otimes_{k} \theta}$ satisfies the following table:

\begin{tabular}{c|c|c|c}
$\omega$ & $L_{1}\left(r_{\theta}\right)$ & $L_{2}\left(r_{\theta}\right)$ & $L_{3}\left(r_{\theta}\right)$ \\
\hline$L_{1}\left(r_{\varphi}\right)$ & $L_{k(1,1)}\left(r_{\varphi \otimes_{k} \theta}\right)$ & $L_{k(1,2)}\left(r_{\varphi \otimes_{k} \theta}\right)$ & $L_{k(1,3)}\left(r_{\varphi \otimes_{k} \theta}\right)$ \\
\hline$L_{2}\left(r_{\varphi}\right)$ & $L_{k(2,1)}\left(r_{\varphi \otimes_{k} \theta}\right)$ & $L_{k(2,2)}\left(r_{\varphi \otimes_{k} \theta}\right)$ & $L_{k(2,3)}\left(r_{\varphi \otimes_{k} \theta}\right)$ \\
\hline$L_{3}\left(r_{\varphi}\right)$ & $L_{k(3,1)}\left(r_{\varphi \otimes_{k} \theta}\right)$ & $L_{k(3,2)}\left(r_{\varphi \otimes_{k} \theta}\right)$ & $L_{k(3,3)}\left(r_{\varphi \otimes_{k} \theta}\right)$
\end{tabular}

Here, again, we have defined $\otimes_{k}$ in a semantical way. Thanks to Theorem 1 , we know that there is a formula in $K_{3}^{S}+\diamond_{1}+\diamond_{2}$ having the ranking function $r_{\varphi \otimes_{k} \theta}$ which is completely described by Table 3 . Thus, $\otimes_{k}$ is well defined up to logical equivalence.

Since the function $k$ determines the operator $\otimes_{k}$, there are $|\{1,2,3\}|^{|\{1,2,3\}|^{2}}=3^{3^{2}}=$ $3^{9}=19,683$ possible belief change operators in this setting. Different functions yield operators with different epistemological attitudes. The Cautious Improvement operator (Definition 1) for instance, gives priority to new information (i.e., to the models of $\theta$ ) but does so in a cautious manner. For example, if an interpretation $\omega$ is a counter model of $\varphi$ and it is a model of $\theta$ then it will be, not a model, but a quasi model of $\varphi * \theta$. Actually, the Cautious Improvement operator is $\otimes_{k}$ when $k$ is defined as follows:

$$
\begin{array}{lll}
k(1,1)=1 & k(1,2)=2 & k(1,3)=2 \\
k(2,1)=1 & k(2,2)=2 & k(2,3)=3 \\
k(3,1)=2 & k(3,2)=2 & k(3,3)=3
\end{array}
$$

\subsection{Examples of Operators Definable in this Framework}

We have already seen that the Cautious Improvement operator is a particular case of these general operators. In the sequel we will see other examples.

The absolute priority operator (or drastic operator) is the operator satisfying the following equality

$$
\varphi \circ \theta=\theta
$$

This operator has the form $\otimes_{k}$ where $k$ is the function: $k(i, j)=j$, thus the table defining the absolute priority operator is is the following one:

\begin{tabular}{c|c|c|c}
$\omega$ & $L_{1}\left(r_{\theta}\right)$ & $L_{2}\left(r_{\theta}\right)$ & $L_{3}\left(r_{\theta}\right)$ \\
\hline$L_{1}\left(r_{\varphi}\right)$ & $L_{1}\left(r_{\varphi \circ \theta}\right)$ & $L_{2}\left(r_{\varphi \circ \theta}\right)$ & $L_{3}\left(r_{\varphi \circ \theta}\right)$ \\
\hline$L_{2}\left(r_{\varphi}\right)$ & $L_{1}\left(r_{\varphi \circ \theta}\right)$ & $L_{2}\left(r_{\varphi \circ \theta}\right)$ & $L_{3}\left(r_{\varphi \circ \theta}\right)$ \\
\hline$L_{3}\left(r_{\varphi}\right)$ & $L_{1}\left(r_{\varphi \circ \theta}\right)$ & $L_{2}\left(r_{\varphi \circ \theta}\right)$ & $L_{3}\left(r_{\varphi \circ \theta}\right)$
\end{tabular}


Contrary to the attitude of the drastic operator, we have the recalcitrant operator which completely ignores the new information. This operator is defined by the following equation:

$$
\varphi \bullet \theta=\varphi
$$

This operator also is in the form $\otimes_{k}$ where $k$ is the function: $k(i, j)=i$, that is, the table defining the recalcitrant operator is the following one:

\begin{tabular}{c|c|c|c}
$\omega$ & $L_{1}\left(r_{\theta}\right)$ & $L_{2}\left(r_{\theta}\right)$ & $L_{3}\left(r_{\theta}\right)$ \\
\hline$L_{1}\left(r_{\varphi}\right)$ & $L_{1}\left(r_{\varphi \bullet \theta}\right)$ & $L_{1}\left(r_{\varphi \bullet \theta}\right)$ & $L_{1}\left(r_{\varphi \bullet \theta}\right)$ \\
\hline$L_{2}\left(r_{\varphi}\right)$ & $L_{2}\left(r_{\varphi \bullet \theta}\right)$ & $L_{2}\left(r_{\varphi \bullet \theta}\right)$ & $L_{2}\left(r_{\varphi \bullet \theta}\right)$ \\
\hline$L_{3}\left(r_{\varphi}\right)$ & $L_{3}\left(r_{\varphi \bullet \theta}\right)$ & $L_{3}\left(r_{\varphi \bullet \theta}\right)$ & $L_{3}\left(r_{\varphi \bullet \theta}\right)$
\end{tabular}

We can also have an operator which is close to contraction via an adequate function $k$. Remember that the postulate of success for a contraction operator can be expressed as follows

$$
\top \forall \theta \Longrightarrow \varphi \ominus \theta \nvdash \theta
$$

that is, if the new piece of information is not a tautology then, after contraction, the resulting formula does not entail the new information.

Define $k:\{1,2,3\}^{2} \longrightarrow\{1,2,3\}$ by putting

$$
k(i, j)= \begin{cases}i & \text { if } j=1 \\ 1 & \text { otherwise }\end{cases}
$$

We call the quasi-contraction operator, denoted $\ominus$, the operator $\otimes_{k}$ using the previously defined $k$. The table defining this operator is the following one:

\begin{tabular}{c|c|c|c}
$\omega$ & $L_{1}\left(r_{\theta}\right)$ & $L_{2}\left(r_{\theta}\right)$ & $L_{3}\left(r_{\theta}\right)$ \\
\hline$L_{1}\left(r_{\varphi}\right)$ & $L_{1}\left(r_{\varphi \ominus \theta}\right)$ & $L_{1}\left(r_{\varphi \ominus \theta}\right)$ & $L_{1}\left(r_{\varphi \ominus \theta}\right)$ \\
\hline$L_{2}\left(r_{\varphi}\right)$ & $L_{2}\left(r_{\varphi \ominus \theta}\right)$ & $L_{1}\left(r_{\varphi \ominus \theta}\right)$ & $L_{1}\left(r_{\varphi \ominus \theta}\right)$ \\
\hline$L_{3}\left(r_{\varphi}\right)$ & $L_{3}\left(r_{\varphi \ominus \theta}\right)$ & $L_{1}\left(r_{\varphi \ominus \theta}\right)$ & $L_{1}\left(r_{\varphi \ominus \theta}\right)$
\end{tabular}

What this table is expressing is that if there are interpretations which are not accepted by the new information, then they will be accepted after contraction. Therefore, the new information will not be entailed after contraction. Thus, the success postulate for the quasicontraction operator is satisfied.

Let us see three graphical examples of this operator at work:

In Figure 2 we can observe that $\varphi \vdash \theta, \theta$ is not a tautology and $\theta$ is not entailed by the formula after contraction. Thus, we see that the success postulate for contraction holds in this example.

The following example (Figure 3) shows the behavior of the operator when the formula to be contracted is a tautology:

In Figure 3 we can observe that $\varphi \vdash \top$ is equal to $\varphi$. Thus the old information remains unchanged when the new information is a tautology.

Example in Figure 4 shows a case when the the information to be contracted is not entailed by the old beliefs. 


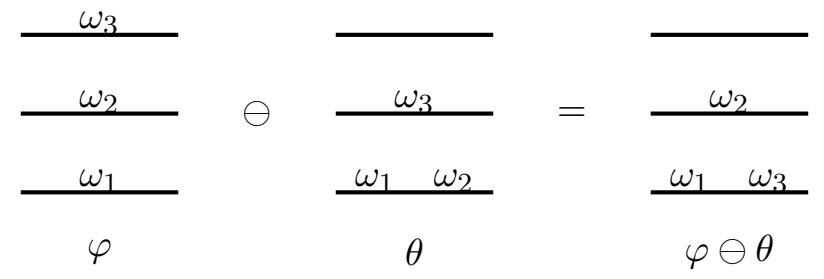

Figure 2: First example of quasi-contraction at work.

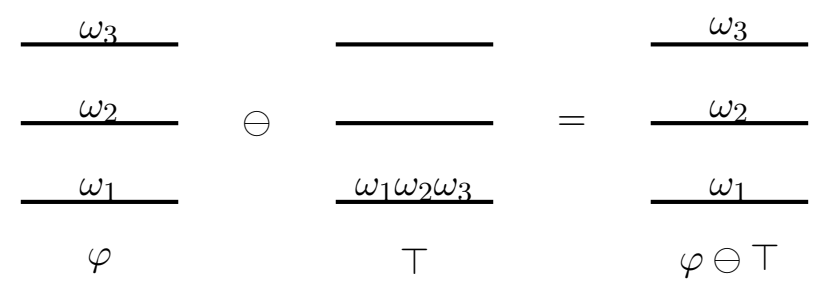

Figure 3: Second example of quasi-contraction at work.

In Figure 4 we can observe that $\varphi \ominus \theta$ is not $\varphi$ even when $\varphi \nvdash \theta$. This example shows that sometimes the quasi-contraction operator produces changes even when the new information is not entailed by the old information. Thus, the postulate of minimality for contraction which establishes that the old information remains unchanged when the new information is not entailed by the old beliefs, is not satisfied.

\subsection{Characterization of General Operators}

We use in this section the same ideas we develop in Section 3.2, that is, we exploit the information given in Table 3 and the techniques developed in Section 2 to find formulas describing the levels of a given formula.

In order to do this, we need to define a set of formulas

$$
\left\{\zeta_{i j}^{l}: i, j, l \in\{1,2,3\}\right\}
$$

as follows. For every triple $(i, j, l)$ :

1. If $l \neq k(i, j)$ then $\zeta_{i j}^{l}=\perp$.

2. If $l=k(i, j)$ then

$$
\zeta_{i j}^{l}=\alpha_{i}^{l} \wedge \beta_{j}^{l}
$$

where

$$
\alpha_{i}^{l}= \begin{cases}\varphi & \text { if } i=1 \\ \square_{1} \varphi \wedge \square_{1} \neg \varphi & \text { if } i=2 \\ \neg \varphi & \text { if } i=3\end{cases}
$$

and

$$
\beta_{j}^{l}= \begin{cases}\theta & \text { if } j=1 \\ \square_{1} \theta \wedge \square_{1} \neg \theta & \text { if } j=2 \\ \neg \theta & \text { if } j=3\end{cases}
$$




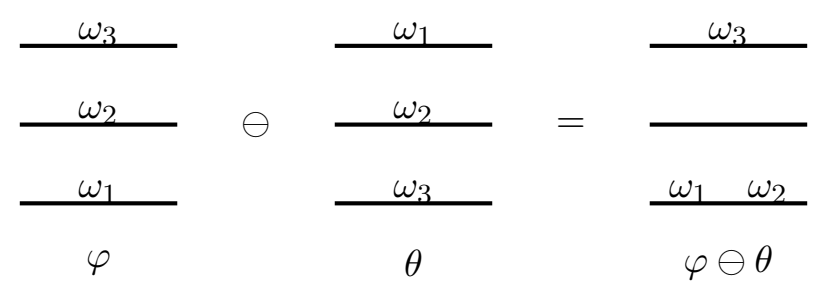

Figure 4: Third example of quasi-contraction at work.

Observe that for any interpretation $\omega$, regardless the value of $l, \omega\left(\alpha_{i}^{l}\right)=1$ iff $\omega \in L_{i}(\varphi)$ and $\omega\left(\beta_{j}^{l}\right)=1$ iff $\omega \in L_{j}(\theta)$.

Note also that if $k(i, j)=l, \omega \in L_{i}(\varphi)$ and $\omega \in L_{j}(\theta)$ then $\omega$ should be in $L_{l}\left(\varphi \otimes_{k} \theta\right)$. This is, actually, the behavior captured by the following postulates:

$$
\begin{gathered}
\bigvee_{1 \leq i \leq j \leq 3} \zeta_{i j}^{1} \exists \equiv(\varphi * \theta) \\
\bigvee_{1 \leq i \leq j \leq 3} \zeta_{i j}^{2} \exists \equiv \square_{1}(\varphi * \theta) \wedge \square_{1} \neg(\varphi * \theta) \\
\bigvee_{1 \leq i \leq j \leq 3} \zeta_{i j}^{3} \exists \models \neg(\varphi * \theta)
\end{gathered}
$$

The postulate $\otimes_{k} 1$ says that the interpretations accepted by $\varphi \otimes_{k} \theta$ are precisely the interpretations satisfying one of the formulas $\zeta_{i j}^{1}$, that is, necessarily, when $k(i, j)=1$, $\omega \in L_{i}(\varphi)$ and $\omega \in L_{j}(\theta)$.

The postulate $\otimes_{k} 2$ says that the interpretations which are uncertain for $\varphi \otimes_{k} \theta$ are precisely the interpretations satisfying one of the formulas $\zeta_{i j}^{2}$, that is, necessarily, when $k(i, j)=2, \omega \in L_{i}(\varphi)$ and $\omega \in L_{j}(\theta)$.

The postulate $\otimes_{k}$ says that the interpretations rejected by $\varphi \otimes_{k} \theta$ are precisely the interpretations satisfying one of the formulas $\zeta_{i j}^{3}$, that is, necessarily, when $k(i, j)=3$, $\omega \in L_{i}(\varphi)$ and $\omega \in L_{j}(\theta)$.

Now we are able to give a syntactic characterization of $\otimes_{k}$.

Theorem 6. Given a function $k:\{1,2,3\}^{2} \longrightarrow\{1,2,3\}$, the operator $\otimes_{k}$ is completely characterized by Postulates $\otimes_{k} 1, \otimes_{k} 2$ and $\otimes_{k} 3$.

Observation 4. A three level partition is completely determined when two of the levels are known, because the interpretations in the third level are exactly the complementary of the union of the other two levels.

By virtue of the previous observation, we have, in a straightforward manner, the following corollary:

Corollary 2. Let $\{i, j\}$ be any pair in $\{1,2,3\}$. The operator $\otimes_{k}$ is completely determined by $\otimes_{k} i$ and $\otimes_{k} j$.

As a matter of fact, one instance of this Corollary, when $k:\{1,2,3\}^{2} \longrightarrow\{1,2,3\}$ is the function associated to the Cautious Improvement operator (see page 12) and $i=1$ and $j=3$, is that the Cautious Improvement operator is characterized by $\otimes_{k} 1$ and $\otimes_{k} 3$, that is to say CI1' and CI2'. 


\section{Related Works}

There are some interesting works linking modal logic and belief revision. Namely, the works of Giacomo Bonanno (Bonanno, 2005, 2007). The aim of these works is to give modal logics in which the process of revision can be simulated and the original postulates of the AGM framework are satisfied. Our aim is different in at least two aspects. First, the processes of change we want to capture in our model differ from the changes proposed in the AGM or KM (Katsuno-Mendelzon) framework where the beliefs before the change (and after it) are represented either as a classical propositional theory or as a classical propositional formula. We model change in a bit more complex structures. The purpose of the 3 -valued logic with modalities we use is to be able to represent these structures through the formulas of the logic and the semantics associated. The second aspect in which our approach differs from those works is in our postulates, which come naturally from the complex process we want to model, that is to say, changes in ranking functions. We may say that our approach to the syntactic postulates is oriented by semantics and this leads to the discovery of the axioms.

It is worth to note that our modalities of type one $\left(\nabla_{1}\right.$ and $\left.\square_{1}\right)$ are related to ideas on improvement operators (Konieczny \& Pino Pérez, 2008; Konieczny et al., 2010; Medina Grespan \& Pino Pérez, 2013). Actually, $\square_{1}$ is similar to the operator of one improvement introduced in those works: the effect of this modality consists of improving by one degree the plausibility of all worlds, whenever possible. Of course, our structures of three levels do not allow to simulate completely operators as the one-improvement operator, because these operators can create new levels in the process of revision. In order to simulate that, we should have structures with at least $3^{n}$ levels, the maximal length of a total preorder on $\mathcal{I}_{n}$.

Note that Hans Rott in his work Shifting Priorities: Simple Representations for TwentySeven Iterated Theory Change Operators (Rott, 2009) analyses the behavior of 27 change operators looking at the changes in spheres' systems (alias total preorders). Our work is reminiscent of this approach and the subtitle of our work is a sort of tribute to his work.

\section{Final Remarks and Future Research}

This work is a first step in order to have a logic in which formulas represent complex epistemic states, such as ranking functions on interpretations, a generalization of total preorders. We have focused here on ranking functions taking three values: acceptation, rejection and indetermination. Mainly, there are three contributions in this work which we want to remark here:

1. We have defined a modal expansion of the Kleene's strong 3-valued logic that allows us to describe three-level preorders associated with any formula $\varphi$ in this logic. Level $L_{1}(\varphi), L_{2}(\varphi)$ and $L_{3}(\varphi)$ are respectively the sets of models, quasi models and countermodels of $\varphi$.

2. We have defined semantically all the change operators $\left(3^{9}\right)$ and, what is most important, we have introduced a technique to characterize them syntactically in the Kleene's strong 3-valued logic with modalities.

3. We have focused on a particular, natural and meaningful operator, called the Cautious Improvement operator. This operator has been characterized through two postulates. 
4. We have characterized all possible change operators $(19,683$ in total) in the three-level structures following the methodology used for characterizing the Cautious Improvement operator.

The main issues we want to develop next are the following:

- To define a complete proof theory for Kleene's strong 3-valued logic expanded with $\diamond_{1}$ and $\diamond_{2}$.

- To characterize syntactically a class of operators in which the new piece of information does not worsen, that is, the truth value of interpretations after revision is at least as good as the truth value of the new information. In symbols, operators satisfying the following inequality: $\omega(\varphi * \theta) \geq \omega(\theta)$, for every interpretation $\omega$. This is important because this class corresponds to operators of general improvement in the logic $K_{3}^{S}+$ $\diamond_{1}+\diamond_{2}$. Note that the Cautious Improvement operator is not in this class.

- To understand when the operators have a representation in terms of the inputs, that is, when the formula corresponding to $\varphi * \theta$ can be expressed in terms of $\varphi$ and $\theta$ within the logic $K_{3}^{S}+\diamond_{1}+\diamond_{2}$.

- In particular, it is for us an open question if the Cautious Improvement operator can be expressed as a formula of the logic $K_{3}^{S}+\diamond_{1}+\diamond_{2}$ in terms of its inputs. We conjecture, even in presence of Theorem 1, that this is impossible.

- To generalize the Kleene's strong 3-valued logic with modalities to a modal $3^{n}$-valued logic which can capture all the ranking functions into $3^{n}$ values and find the way to encode in such a logic important attitudes with respect to the new information, such as acceptance, rejection, improvement and some of its variants. Note that in the DP approach to revision (Darwiche \& Pearl, 1997) (a generalization of AGM operators) there is in general creation of levels in the output. Thus, it is not at all clear if this kind of operators can be encoded in a natural way in a framework with ranking functions where the number of possible values is fixed.

- To explore the links between our operators and non monotonic logics, exploiting the ideas of duality between belief revision and the rational relations (see Freund \& Lehmann, 2002; Gärdenfors, 1991; Makinson \& Gärdenfors, 1991). The idea is, given a formula $\varphi$ and an operator $*$, to define the entailment $\mid \sim$ by putting $\alpha \mid \sim \beta$ if, and only if, $\varphi * \alpha \vdash \beta$. Then, the program is to study the logical properties of this kind of entailment.

\section{Acknowledgments}

We thank the anonymous reviewers for their careful reading and their clever remarks which certainly have helped us a lot to improve the presentation and the quality of this work.

The second author thanks the Consejo de Desarrollo Cientifico Humanistico Tecnológico y de las Artes de la Universidad de Los Andes (CDCHTA-ULA) for its partial support through the Project $\mathrm{N}^{\circ} \mathrm{C}-1855-13-05-\mathrm{AA}$.

Finally, we thank our colleague Professor Olga Porras for the careful English proofreading. 


\section{Appendix A. Proofs}

Proof of Theorem 1: Let $r: \mathcal{I}_{n} \longrightarrow\left\{1, \frac{1}{2}, 0\right\}$ be a ranking function. We know, due to Equation 1, that there are formulas $\psi_{1}, \psi_{2}$ and $\psi_{3}$ such that $\omega\left(\psi_{j}\right)=1$ if and only if $\omega \in L_{j}(r)$. Define the formula ${ }^{2}$

$$
\varphi_{r}:=\neg\left(\diamond_{1} \psi_{2} \vee \diamond_{2} \psi_{3}\right)
$$

Then,

$$
\begin{aligned}
\omega \in L_{1}(r) & \Longleftrightarrow \omega \notin L_{2}(r) \quad \text { and } \omega \notin L_{3}(r) \\
& \Longleftrightarrow \omega\left(\psi_{2}\right), \omega\left(\psi_{3}\right) \in\{0,1 / 2\} \\
& \Longleftrightarrow \omega\left(\diamond_{1} \psi_{2}\right)=\omega\left(\diamond_{2} \psi_{3}\right)=0 \\
& \Longleftrightarrow \omega\left(\diamond_{1} \psi_{2} \vee \diamond_{2} \psi_{3}\right)=0 \\
& \Longleftrightarrow \omega\left(\neg\left(\diamond_{1} \psi_{2} \vee \diamond_{2} \psi_{3}\right)\right)=1
\end{aligned}
$$

i.e.,

$$
\omega \in L_{1}(r) \Longleftrightarrow \omega\left(\varphi_{r}\right)=1
$$

For the characterization of the second level, we have

$$
\begin{aligned}
\omega \in L_{2}(r) & \Longrightarrow \omega\left(\psi_{2}\right)=1 \quad \text { and } \omega\left(\psi_{3}\right) \in\{0,1 / 2\} \\
& \Longrightarrow \omega\left(\diamond_{1} \psi_{2}\right)=1 / 2 \text { and } \omega\left(\diamond_{2} \psi_{3}\right)=0 \\
& \Longrightarrow \omega\left(\diamond_{1} \psi_{2} \vee \diamond_{2} \psi_{3}\right)=1 / 2 \\
& \Longrightarrow \omega\left(\neg\left(\diamond_{1} \psi_{2} \vee \diamond_{2} \psi_{3}\right)\right)=1 / 2
\end{aligned}
$$

On the other hand,

$$
\begin{aligned}
\omega\left(\neg\left(\diamond_{1} \psi_{2} \vee \diamond_{2} \psi_{3}\right)\right)=1 / 2 & \Longrightarrow \omega\left(\diamond_{1} \psi_{2} \vee \diamond_{2} \psi_{3}\right)=1 / 2 \\
& \Longrightarrow \omega\left(\diamond_{1} \psi_{2}\right)=1 / 2 \quad \text { or } \quad \omega\left(\diamond_{2} \psi_{3}\right)=1 / 2
\end{aligned}
$$

It is not possible to have $\omega\left(\diamond_{2} \psi_{3}\right)=1 / 2$ because the truth value of $\diamond_{2} \psi_{3}$ is either 1 or 0 under any interpretation, thus, it must be $\omega\left(\nabla_{1} \psi_{2}\right)=1 / 2$, so $\omega\left(\psi_{2}\right)=1$ and as a consequence $\omega \in L_{2}(r)$.

Hence

$$
\omega \in L_{2}(r) \Longleftrightarrow \omega\left(\varphi_{r}\right)=1 / 2
$$

for any interpretation $\omega$.

Finally, for the third level:

$$
\begin{aligned}
\omega \in L_{3}(r) & \Longrightarrow \omega\left(\psi_{2}\right) \in\{0,1 / 2\} \quad \text { and } \omega\left(\psi_{3}\right)=1 \\
& \Longrightarrow \omega\left(\diamond_{1} \psi_{2}\right)=0 \text { and } \omega\left(\diamond_{2} \psi_{3}\right)=1 \\
& \Longrightarrow \omega\left(\diamond_{1} \psi_{2} \vee \diamond_{2} \psi_{3}\right)=1
\end{aligned}
$$

2. Note that in formula $\varphi_{r}$, formula $\psi_{1}$ doesn't appear as a parameter. This is, essentially, due to the fact that knowing two levels of the partition leads to knowing the third level. Actually, the formula $\varphi_{r}$ is not unique. 


$$
\Longrightarrow \omega\left(\neg\left(\diamond_{1} \psi_{2} \vee \diamond_{2} \psi_{3}\right)\right)=0
$$

For the converse, we have

$$
\omega\left(\neg\left(\diamond_{1} \psi_{2} \vee \diamond_{2} \psi_{3}\right)\right)=0 \Longrightarrow \omega\left(\diamond_{1} \psi_{2} \vee \diamond_{2} \psi_{3}\right)=1
$$

Thus, either $\omega\left(\nabla_{1} \psi_{2}\right)=1$ or $\omega\left(\nabla_{2} \psi_{3}\right)=1$. Since $\omega\left(\diamond_{1} \theta\right) \leq 1 / 2$ for every formula $\theta$, it must be the case that $\omega\left(\diamond_{2} \psi_{3}\right)=1$, so $\omega\left(\psi_{3}\right)=1$ and $\omega \in L_{3}(r)$. Consequently

$$
\omega \in L_{3}(r) \Longleftrightarrow \omega\left(\varphi_{r}\right)=0
$$

Henceforth $\varphi_{r}$ captures completely the ranking function $r$.

Proof of Theorem 2: We consider $K_{3}^{S}+\diamond_{i}$ for $i=1,2$ with only one variable. We have the interpretations $\omega_{1}, \omega_{2}, \omega_{3}$ such that $\omega_{1}(x)=1, \omega_{2}(x)=1 / 2$ and $\omega_{3}(x)=0$. Consider the set $\mathcal{P}=\left\{P_{0}, P_{1}, \ldots, P_{26}\right\}$ of all three-level preorders on these three interpretations, being $P_{0}$ the preorder

$$
\begin{aligned}
& \omega_{3} \\
& \omega_{2} \\
& \omega_{1}
\end{aligned}
$$

This is represented by the formula $\varphi_{0}:=x$ in the sense that $\omega_{1}\left(\varphi_{0}\right)=1, \omega_{2}\left(\varphi_{0}\right)=1 / 2$ and $\omega_{3}\left(\varphi_{0}\right)=0$.

1. In order to prove this result for $K_{3}^{S}+\diamond_{1}$, we define the operations $\neg, \square_{1}$ and $\vee$ on the set of preorders. Given preorders $P, P^{\prime}$ :

(a) $L_{1}(\neg P)=L_{3}(P), L_{2}(\neg P)=L_{2}(P)$ and $L_{3}(\neg P)=L_{1}(P)$.

(b) $L_{1}\left(\square_{1} P\right)=L_{1}(P) \cup L_{2}(P), L_{2}\left(\square_{1} P\right)=L_{3}(P)$ and $L_{3}\left(\square_{1} P\right)=\emptyset$.

(c) If interpretation $\omega$ is at level $L_{i}(P)$ and level $L_{j}\left(P^{\prime}\right)$, then $\omega$ is at level $L_{k}\left(P \vee P^{\prime}\right)$ where $k=\min \{i, j\}$.

Let us call ${\overline{P_{0}}}^{1}$ the closure of the set $\left\{P_{0}\right\}$ under these three operations and ${\overline{P_{0 k}}}^{1}$ the set of the preorders obtained after $k$ or less successive applications of $\left\{\neg, \square_{1}, \vee\right\}$.

Let us say the preorder $P$ belongs to the set $\mathcal{F}_{1}$ if and only if it has one of the following forms:

$\begin{array}{cccc}\omega & \omega_{2} & \omega & \omega^{\prime} \omega^{\prime \prime} \\ \omega^{\prime} & \omega^{\prime} & \emptyset & \emptyset \\ \omega_{2} & \omega & \omega^{\prime} \omega^{\prime \prime} & \omega\end{array}$

We will prove that no preorder in $\mathcal{F}_{1}$ belongs to ${\overline{P_{0}}}^{1}$. We do it by induction in the number of steps, being our base case the application of the operations to $P_{0}$. These yield:

$$
\neg P_{0}=\begin{gathered}
\omega_{1} \\
\omega_{2} \\
\omega_{3}
\end{gathered} \quad \square_{1} P_{0}=\begin{gathered}
\emptyset \\
\omega_{3} \\
\omega_{1} \omega_{2}
\end{gathered} \quad P_{0} \vee P_{0}=P_{0}
$$


Obviously none of those preorders belongs to $\mathcal{F}_{1}$.

Now suppose that no preorder in $\mathcal{F}_{1}$ belongs to ${\overline{P_{0}}}_{k}^{1}$.

Given a sequence $P_{i_{0}}, P_{i_{1}}, \ldots, P_{i_{k}}, P_{i_{k+1}}$ of preorders with $i_{0}=0$ such that $P_{i_{j+1}}$ is obtained from $P_{i_{j}}$ by an application of one of our operators, suppose, for a contradiction, that $P_{i_{k+1}}$ is in $\mathcal{F}_{1}$. Then:

(a) If $P_{i_{k+1}}=\neg P_{i_{k}}$ then $P_{i_{k}}$ must be in $\mathcal{F}_{1}$ which is a contradiction.

(b) If $P_{i_{k+1}}=\square_{1} P_{i_{k}}$ then $L_{3}\left(P_{i_{k+1}}\right)$ is empty thus it is not a linear order and it has a level which is empty other than $L_{2}\left(P_{i_{k+1}}\right)$ hence $P_{i_{k+1}}$ is not in $\mathcal{F}_{1}$.

(c) If $P_{i_{k+1}}=P_{i_{k}} \vee P^{\prime}$ with $P^{\prime}$ a preorder in ${\overline{P_{0}}}_{k}^{1}$ :

- Suppose $P_{i_{k+1}}$ is a linear order with $\omega_{2}$ in $L_{3}$, i.e.,

$$
P_{i_{k+1}}=\begin{gathered}
\omega_{2} \\
\omega \\
\omega^{\prime}
\end{gathered}
$$

then $\omega_{2}$ must be in both $L_{3}\left(P_{i_{k}}\right)$ and $L_{3}\left(P^{\prime}\right)$ and $\omega^{\prime}$ must be in $L_{1}\left(P_{i_{k}}\right)$ or $L_{1}\left(P^{\prime}\right)$. Without loss of generality, we may assume that $\omega_{2} \in L_{3}\left(P^{\prime}\right)$ and $\omega^{\prime} \in L_{1}\left(P^{\prime}\right)$. Then necessarily either $\omega \in L_{2}\left(P^{\prime}\right)$ and $P^{\prime}=P_{i_{k+1}}$ or $L_{2}\left(P^{\prime}\right)=\emptyset$ in $P^{\prime}$. In both cases, we can conclude that $P^{\prime} \in \mathcal{F}_{1}$.

- Similarly, if $P_{i_{k+1}}$ is a linear order with $\omega_{2}$ in $L_{1}$

$$
P_{i_{k+1}}=\begin{gathered}
\omega \\
\omega^{\prime} \\
\omega_{2}
\end{gathered}
$$

then $\omega$ must be in both $L_{3}\left(P_{i_{k}}\right)$ and $L_{3}\left(P^{\prime}\right)$ and $\omega_{2}$ must be either in $L_{1}\left(P_{i_{k}}\right)$ or $L_{1}\left(P^{\prime}\right)$. Again, suppose without loss of generality that $P^{\prime}$ has $\omega$ in $L_{3}$ and $\omega_{2}$ in $L_{1}$. If $\omega^{\prime}$ is not in $L_{2}$ for $P^{\prime}$ then this level is empty and if $\omega^{\prime}$ is in $L_{2}$ then $P^{\prime}=P_{i_{k+1}}$. In both cases, we have $P^{\prime} \in \mathcal{F}_{1}$.

- If $P_{i_{k+1}}$ has the form

then necessarily:

$$
\begin{gathered}
\omega \\
\emptyset \\
\omega^{\prime} \omega^{\prime \prime}
\end{gathered}
$$

- $\omega$ belongs to level $L_{3}\left(P_{k}\right)$ and $L_{3}\left(P^{\prime}\right)$.

- $\omega^{\prime}$ must be either in $L_{1}\left(P_{k}\right)$ or $L_{1}\left(P^{\prime}\right)$.

- $\omega$ must be either in $L_{1}\left(P_{k}\right)$ or $L_{1}\left(P^{\prime}\right)$.

Suppose that $\omega^{\prime}$ and $\omega^{\prime \prime}$ are in $L_{1}\left(P^{\prime}\right)$, then

$$
P^{\prime}=\begin{gathered}
\omega \\
\emptyset \\
\omega^{\prime} \omega^{\prime \prime}
\end{gathered}
$$

thus $P^{\prime} \in \mathcal{F}_{1}$. If this is not the case then necessarily

$$
P^{\prime}=\begin{gathered}
\omega \\
\omega^{\prime} \\
\omega^{\prime \prime}
\end{gathered} \quad \text { or } \quad P^{\prime}=\begin{gathered}
\omega \\
\omega^{\prime \prime} \\
\omega^{\prime}
\end{gathered}
$$


We examine only the case

$$
P^{\prime}=\begin{aligned}
& \omega \\
& \omega^{\prime} \\
& \omega^{\prime \prime}
\end{aligned}
$$

since the other one is symmetric. In this case, necessarily

$$
P_{k}=\begin{gathered}
\omega \\
\omega^{\prime \prime} \\
\omega^{\prime}
\end{gathered}
$$

then one of those two preorders is linear with $\omega_{2}$ in one of the extreme levels, hence one of them is in $\mathcal{F}_{1}$.

- Finally, suppose that If $P_{i_{k+1}}$ has the form

$$
\begin{gathered}
\omega^{\prime} \omega^{\prime \prime} \\
\emptyset \\
\omega
\end{gathered}
$$

then both $P_{k}$ and $P^{\prime}$ must have $\omega^{\prime}$ and $\omega^{\prime \prime}$ in $L_{3}$ and one of them has to have $\omega$ in $L_{1}$. Suppose that $P^{\prime}$ satisfies these two properties, then $P^{\prime}=P_{i_{k+1}}$ and as a consequence $P^{\prime} \in \mathcal{F}_{1}$.

As we can see, on each case we contradict the inductive hypothesis, thus it is not possible that $P_{i_{k+1}} \in \mathcal{F}_{1}$ without incurring in a contradiction. Hence, $P_{i_{k+1}} \notin \mathcal{F}_{1}$ thus no element of $\mathcal{F}_{1}$ belongs to the closure of $P_{0}$ under the given operations.

As a consequence of this, there are preorders in $\mathcal{P}$ that have no corresponding formula in $K_{3}^{S}+\diamond_{1}$.

2. Now to prove that $K_{3}^{S}+\diamond_{2}$, in the finite case, cannot capture all the preorders, we define a new operator $\square_{2}$ for every preorder $P$ as

$$
\begin{aligned}
& L_{1}\left(\square_{2} P\right)=L_{1}(P) \cup L_{2}(P) \\
& L_{2}\left(\square_{2} P\right)=\emptyset \\
& L_{3}\left(\square_{2} P\right)=L_{3}(P)
\end{aligned}
$$

Let us denote by ${\overline{P_{0}}}^{2}$ the closure of $\left\{P_{0}\right\}$ under operators $\neg, \square_{2}, \vee$ and by ${\overline{P_{0 k}}}^{2}$ the set of the preorders obtained by $k$ or less successive applications of these operators. Let $\mathcal{F}_{2}$ denote the set of the preorders with one of the following configurations:

$\begin{array}{ccccccc}\omega_{2} & \omega & \emptyset & \emptyset & \omega_{2} \omega^{\prime} & \emptyset & \omega^{\prime \prime} \\ \omega^{\prime} & \omega^{\prime} & \omega \omega^{\prime} \omega^{\prime \prime} & \omega & \omega & \omega \omega^{\prime} & \omega \omega^{\prime} \\ \omega & \omega_{2} & \emptyset & \omega_{2} \omega^{\prime} & \emptyset & \omega^{\prime \prime} & \emptyset\end{array}$

We will prove, using induction in the number of steps, that $P \in{\overline{P_{0}}}^{2}$ implies that $P$ is not in $\mathcal{F}_{2}$.

Our base case consists of applying these operators to $P_{0}$. As we saw in Equation 5, neither $\neg P_{0}$ nor $P_{0} \vee P_{0}$ belong to $\mathcal{F}_{2}$. On the other hand

$$
\square_{2} P_{0}=\begin{gathered}
\omega_{3} \\
\emptyset \\
\omega_{1} \omega_{2}
\end{gathered}
$$


which is clearly not in $\mathcal{F}_{2}$.

For the inductive hypothesis, assume that no preorder in ${\overline{P_{0}}}_{k}^{2}$ belongs to $\mathcal{F}_{2}$.

Suppose we have a sequence $P_{i_{0}}, P_{i_{1}}, \ldots, P_{i_{k}}, P_{i_{k+1}}$ of preorders with $i_{0}=0$ such that $P_{i_{j+1}}$ is obtained from $P_{i_{j}}$ by an application of one of our operators and assume for a contradiction that $P_{i_{k+1}}$ is in $\mathcal{F}_{2}$. Hence:

(a) If $P_{i_{k+1}}=\neg P_{i_{k}}$ it is immediate that $P_{i_{k+1}} \in \mathcal{F}_{2}$ iff $P_{i_{k}} \in \mathcal{F}_{2}$.

(b) Suppose $P_{i_{k+1}}=\square_{2} P_{i_{k}}$, then $L_{2}\left(P_{i_{k+1}}\right)$ is empty, thus $P_{i_{k+1}}$ does not belong to $\mathcal{F}_{2}$.

(c) If $P_{i_{k+1}}=P_{i_{k}} \vee P^{\prime}$ with $P^{\prime}$ a preorder in ${\overline{P_{0 k}}}^{2}$ we need to consider seven cases.

i. If $P_{i_{k+1}}$ has the form

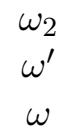

then $\omega_{2}$ belongs to $L_{3}\left(P_{i_{k}}\right)$ and $L_{3}\left(P^{\prime}\right)$ and $\omega$ must be either in $L_{1}\left(P_{i_{k}}\right)$ or $L_{1}\left(P^{\prime}\right)$. If $\omega$ is in $L_{1}\left(P^{\prime}\right)$ then $P^{\prime}$ must have the form

$$
\begin{gathered}
\omega_{2} \omega^{\prime} \\
\emptyset \\
\omega
\end{gathered}
$$

since otherwise it would be in $\mathcal{F}_{2}$. But then $\omega^{\prime}$ must be in $L_{2}\left(P_{i_{k}}\right)$ and as a consequence we obtain that $P_{i_{k}}$ is in $\mathcal{F}_{2}$ wherever we place $\omega$ because $P_{i_{k}}$ would be in one of the following forms

$\begin{array}{ccc}\omega_{2} & \omega_{2} & \omega_{2} \omega \\ \omega^{\prime} & \omega^{\prime} \omega & \omega^{\prime} \\ \omega & \emptyset & \emptyset\end{array}$

ii. If $P_{i_{k+1}}$ is in the form

$$
\begin{gathered}
\omega \\
\omega^{\prime} \\
\omega_{2}
\end{gathered}
$$

then $\omega \in L_{3}\left(P_{i_{k}}\right)$ and $\omega \in L_{3}\left(P^{\prime}\right)$ and $\omega_{2} \in L_{1}\left(P_{i_{k}}\right)$ or $\omega_{2} \in L_{1}\left(P^{\prime}\right)$. Suppose $\omega \in L_{3}\left(P^{\prime}\right)$ and $\omega_{2} \in L_{1}\left(P^{\prime}\right)$, then we must have $\omega^{\prime} \in L_{3}\left(P^{\prime}\right)$ or, otherwise, $P^{\prime}$ belongs to $\mathcal{F}_{2}$ thus $P^{\prime}$ must have the form

$$
\begin{gathered}
\omega \omega^{\prime} \\
\emptyset \\
\omega_{2}
\end{gathered}
$$

But in this case we must have $\omega^{\prime} \in L_{2}\left(P_{i_{k}}\right)$ and this implies that $P_{i_{k}}$ is in one of the following forms:

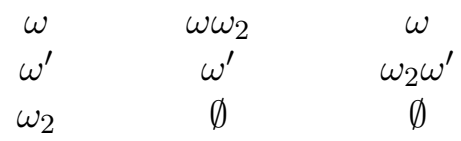

hence $P^{\prime} \notin \mathcal{F}_{2} \Longrightarrow P_{i_{k}} \in \mathcal{F}_{2}$.

iii. Suppose $P_{i_{k+1}}$ has the form 


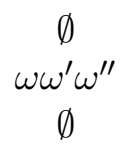

If neither $P_{i_{k}}$ nor $P^{\prime}$ have this form, there are two possible combinations that yield this:

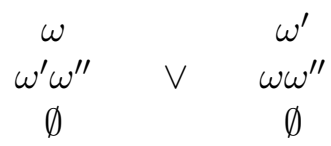

and

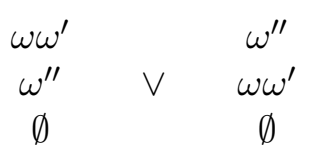

but every preorder in the form

$$
\begin{gathered}
\omega^{\prime \prime} \\
\omega \omega^{\prime} \\
\emptyset
\end{gathered}
$$

belongs to $\mathcal{F}_{2}$.

iv. When $P_{i_{k+1}}$ is in the form

$$
\begin{gathered}
\emptyset \\
\omega \\
\omega_{2}, \omega^{\prime}
\end{gathered}
$$

there are two subcases to consider.

First, suppose without loss of generality that $\omega_{2}$ and $\omega^{\prime}$ belong to $L_{1}\left(P^{\prime}\right)$ then, as $P^{\prime}$ is not in $\mathcal{F}_{2}$, we must have

$$
P^{\prime}=\begin{gathered}
\omega \\
\emptyset \\
\omega_{2} \omega^{\prime}
\end{gathered}
$$

In this case $\omega$ must be at $L_{2}\left(P_{i_{k}}\right)$. If $P_{i_{k}}$ is linear, $\omega_{2}$ is in $L_{1}\left(P_{i_{k}}\right)$ or $L_{3}\left(P_{i_{k}}\right)$ and it is in $\mathcal{F}_{2}$. Thus suppose $P_{i_{k}}$ is not linear, hence it must have $\omega_{2}$ and $\omega^{\prime}$ at the same level and wherever we put $\omega_{2}, \omega^{\prime}$ we obtain that $P_{i_{k}}$ is in $\mathcal{F}_{2}$.

Suppose now, without loss of generality, that $\omega_{2}$ is in $L_{1}\left(P_{i_{k}}\right)$ and $\omega^{\prime}$ is in $L_{1}\left(P^{\prime}\right)$. Observe that $P_{i_{k}}$ cannot be linear in this case and that we already examined the case when $\omega_{2}$ and $\omega^{\prime}$ are both in $L_{1}\left(P_{i_{k}}\right)$, thus we can suppose $\omega$ and $\omega^{\prime}$ are at the same level, which must be $L_{2}$ or $L_{3}$. If $\omega$ and $\omega^{\prime}$ are in $L_{2}\left(P i_{k}\right)$ then

$$
P i_{k}=\begin{gathered}
\emptyset \\
\omega \omega^{\prime} \\
\omega_{2}
\end{gathered}
$$

which belongs to $\mathcal{F}_{2}$. If, on the other hand, $\omega$ and $\omega^{\prime}$ are in $L_{3}\left(P i_{k}\right)$ we have that

$$
P i_{k}=\begin{gathered}
\omega \omega^{\prime} \\
\emptyset \\
\omega_{2}
\end{gathered}
$$

thus $\omega$ is in $L_{2}\left(P^{\prime}\right)$ and $P^{\prime}$ must be in one of the following forms 


$\begin{array}{ccc}\omega^{\prime} & \emptyset & \emptyset \\ \omega & \omega \omega^{\prime} & \omega \\ \omega_{2} & \omega_{2} & \omega_{2} \omega^{\prime}\end{array}$

and all of these forms are in $\mathcal{F}_{2}$.

v. Suppose $P_{i_{k+1}}$ has the form

$$
\begin{gathered}
\omega_{2} \omega^{\prime} \\
\omega \\
\emptyset
\end{gathered}
$$

In this case we must have $\omega_{2}, \omega^{\prime} \in L_{3}\left(P_{i_{k}}\right)$ and $\omega_{2}, \omega^{\prime} \in L_{3}\left(P^{\prime}\right)$ and necessarily either $\omega \in L_{2}\left(P_{i_{k}}\right)$ or $\omega \in L_{2}\left(P^{\prime}\right)$. Thus either $P_{i_{k+1}}=P_{i_{k}}$ or $P_{i_{k+1}}=P^{\prime}$ which is not possible because then $P_{i_{k+1}}$ can be obtained in $k$ steps and it contradicts the inductive hypothesis.

vi. If $P_{i_{k+1}}$ has the form

$$
\begin{gathered}
\omega^{\prime \prime} \\
\omega \omega^{\prime} \\
\emptyset
\end{gathered}
$$

then necessarily $L_{1}\left(P_{i_{k}}\right)=L_{1}\left(P^{\prime}\right)=\emptyset$ and $\omega^{\prime \prime}$ is in $L_{3}\left(P_{i_{k}}\right)$ and $L_{3}\left(P^{\prime}\right)$. If neither $P_{i_{k}}$ nor $P^{\prime}$ belong to $\mathcal{F}_{2}$ the only combination yielding the given configuration is

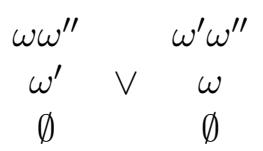

as at least one of them have $\omega_{2}$ at level $L_{3}$, then at least one of them is in $\mathcal{F}_{2}$.

vii. If $P_{i_{k+1}}$ has the form

$$
\begin{gathered}
\emptyset \\
\omega \omega^{\prime} \\
\omega^{\prime \prime}
\end{gathered}
$$

Suppose, without loss of generality, that $\omega, \omega^{\prime} \in L_{2}\left(P^{\prime}\right)$ then necessarily $P^{\prime} \in \mathcal{F}_{2}$ since it must be in one of the forms

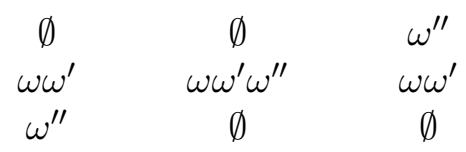

Thus we can assume that $\left\{\omega, \omega^{\prime}\right\} \nsubseteq L_{2}\left(P^{\prime}\right)$ and $\left\{\omega, \omega^{\prime}\right\} \nsubseteq L_{2}\left(P_{i_{k}}\right)$. Since $\left\{\omega, \omega^{\prime}\right\}=L_{2}\left(P_{i_{k+1}}\right)$ it must also be the case that $\left\{\omega, \omega^{\prime}\right\} \nsubseteq L_{3}\left(P^{\prime}\right)$ and $\left\{\omega, \omega^{\prime}\right\} \nsubseteq L_{3}\left(P_{i_{k}}\right)$ because if, for instance, we have that $\left\{\omega, \omega^{\prime}\right\} \subseteq L_{3}\left(P^{\prime}\right)$ then we need $\left\{\omega, \omega^{\prime}\right\} \subseteq L_{2}\left(P_{i_{k}}\right)$.

We have a case with $\omega \in L_{3}\left(P_{i_{k}}\right), \omega^{\prime} \in L_{2}\left(P_{i_{k}}\right), \omega \in L_{2}\left(P^{\prime}\right)$ and $\omega^{\prime} \in$ $L_{3}\left(P^{\prime}\right)$ (the other possible case is symmetric) but then necessarily either $\omega^{\prime \prime} \in L_{1}\left(P_{i_{k}}\right)$ or $\omega^{\prime \prime} \in L_{1}\left(P^{\prime}\right)$. Suppose $\omega^{\prime \prime} \in L_{1}\left(P^{\prime}\right)$ (the other case is, again, symmetric) thus we have one of the following cases: 
One possibility is

$$
P_{i_{k+1}}=\begin{array}{ccc}
\omega^{\prime} & & \omega \\
\omega & \vee & \omega^{\prime} \\
\omega^{\prime \prime} & & \omega^{\prime \prime}
\end{array}
$$

in this case, we have at least one linear order with $\omega_{2}$ at an extreme level, hence at least one of the preorders is in $\mathcal{F}_{2}$.

Another possible configuration is

$$
P_{i_{k+1}}=\begin{array}{ccc}
\omega^{\prime} & & \omega \\
\omega & \vee & \omega^{\prime} \omega^{\prime \prime} \\
\omega^{\prime \prime} & & \emptyset
\end{array}
$$

but in this case $P_{i_{k}} \in \mathcal{F}_{2}$.

The remaining possibility is

$$
P_{i_{k+1}}=\begin{array}{ccc}
\omega^{\prime} & & \omega \omega^{\prime \prime} \\
\omega & \vee & \omega^{\prime} \\
\omega^{\prime \prime} & & \emptyset
\end{array}
$$

If $\omega^{\prime} \neq \omega_{2}$ and $\omega^{\prime \prime} \neq \omega_{2}$ then $\omega=\omega_{2}$ and

$$
P_{i_{k}}=\begin{gathered}
\omega_{2} \omega^{\prime \prime} \\
\omega^{\prime} \\
\emptyset
\end{gathered} \in \mathcal{F}_{2}
$$

and this finishes the proof.

Proof of Theorem 3: According to CI1',

$$
(\varphi \wedge \theta) \vee\left(\square_{1} \varphi \wedge \square_{1} \neg \varphi \wedge \theta\right)=\vDash \varphi * \theta
$$

It is easy to check, using truth tables, that

$$
(\varphi \wedge \theta) \vee\left(\square_{1} \varphi \wedge \square_{1} \neg \varphi \wedge \theta\right)=\vDash\left(\varphi \vee\left(\square_{1} \varphi \wedge \square_{1} \neg \varphi\right)\right) \wedge \theta
$$

and

$$
\left(\varphi \vee\left(\square_{1} \varphi \wedge \square_{1} \neg \varphi\right)\right)=\equiv \square_{1} \varphi
$$

hence

$$
\varphi * \theta=\equiv \square_{1} \varphi \wedge \theta
$$

That is CI1.

On the other hand, by CI2'

$$
\left(\left(\square_{1} \varphi \wedge \square_{1} \neg \varphi\right) \wedge \neg \theta\right) \vee(\neg \varphi \wedge \neg \theta)=\neg(\varphi * \theta)
$$

And again, using truth tables, it is easy to check that

$$
\left(\square_{1} \varphi \wedge \square_{1} \neg \varphi \wedge \neg \theta\right) \vee(\neg \varphi \wedge \neg \theta)=\left(\left(\square_{1} \varphi \wedge \square_{1} \neg \varphi\right) \vee \neg \varphi\right) \wedge \neg \theta
$$


and

$$
\varphi \vee\left(\square_{1} \varphi \wedge \square_{1} \neg \varphi\right)=\equiv \square_{1} \neg \varphi
$$

thus

$$
\neg(\varphi * \theta)=\vDash \square_{1} \neg \varphi \wedge \neg \theta
$$

That is CI2.

Proof of Theorem 4: It is a consequence of Theorem 3 and the following two lemmas:

Lemma 1. The Cautious Improvement operator satisfies the postulates CI1' and CI2'

Proof: CI1': If $\omega\left((\varphi \wedge \theta) \vee\left(\left(\square_{1} \varphi \wedge \square_{1} \neg \varphi\right) \wedge \theta\right)\right)=1$ then either $\omega(\varphi \wedge \theta)=1$ or $\omega\left(\left(\square_{1} \varphi \wedge \square_{1} \neg \varphi\right) \wedge \theta\right)=1$. In the first case, $\omega(\varphi)=\omega(\theta)=1$ thus $\omega \in L_{1}(\varphi) \cap L_{1}(\theta)$ and this implies by Table 2 that $\omega \in L_{1}(\varphi * \theta)$, and so $\omega(\varphi * \theta)=1$.

In the second case, we have $\omega\left(\square_{1} \varphi \wedge \square_{1} \neg \varphi\right)=\omega(\theta)=1$ which implies that $\omega \in L_{1}(\theta)=1$. On the other hand,

$$
\omega\left(\square_{1} \varphi\right)=1 \Longrightarrow \omega(\varphi) \geq 1 / 2
$$

and

$$
\begin{aligned}
\omega\left(\square_{1} \neg \varphi\right)=1 & \Longrightarrow \omega(\neg \varphi) \geq 1 / 2 \\
& \Longrightarrow \omega(\varphi) \leq 1 / 2
\end{aligned}
$$

then

$$
1 / 2 \leq \omega(\varphi) \leq 1 / 2
$$

thus

$$
1 / 2 \leq \omega(\varphi)=1 / 2
$$

and $\omega \in L_{2}(\varphi)$. Hence $\omega \in L_{1}(\varphi * \theta)$ by Table 2 and $\omega(\varphi * \theta)=1$.

Conversely, if $\omega(\varphi * \theta)=1$ then $\omega \in L_{1}(\varphi * \theta)$. There are only two cases in Table 2 that make this possible: either $\omega \in L_{1}(\varphi) \cap L_{1}(\theta)$ or $\omega \in L_{2}(\varphi) \cap L_{1}(\theta)$.

If $\omega \in L_{1}(\varphi) \cap L_{1}(\theta)$ then $\omega(\varphi)=\omega(\theta)=1$ and $\omega(\varphi \wedge \theta)=1$. If $\omega \in L_{2}(\varphi) \cap L_{1}(\theta)$ then $\omega\left(\square_{1} \varphi \wedge \square_{1} \neg \varphi\right)=1$ and $\omega(\theta)=1$, hence $\omega\left(\left(\square_{1} \varphi \wedge \square_{1} \neg \varphi\right) \theta\right)=1$. Thus if $\omega(\varphi * \theta)=1$ we have that $\omega\left((\varphi \wedge \theta) \vee\left(\left(\square_{1} \varphi \wedge \square_{1} \neg \varphi\right) \wedge \theta\right)\right)=1$.

CI2': Suppose $\omega$ is an interpretation such that $\omega\left(\left(\left(\square_{1} \varphi \wedge \square_{1} \neg \varphi\right) \wedge \neg \theta\right) \vee(\neg \varphi \wedge \neg \theta)\right)=1$. Then either $\omega\left(\left(\left(\square_{1} \varphi \wedge \square_{1} \neg \varphi\right) \wedge \neg \theta\right)\right)=1$ or $\omega(\neg \varphi \wedge \neg \theta)=1$. In both cases $\omega(\neg \theta)=1$ which implies that $\omega \in L_{3}(\theta)$. In the first case, $\omega\left(\square_{1} \varphi \wedge \square_{1} \neg \varphi\right)=1$ implies that $\omega \in L_{2}(\varphi)$ thus $\omega \in L_{2}(\varphi) \cap L_{3}(\theta)$. In the second case, $\omega(\neg \theta)=1$ implies that $\omega \in L_{3}(\varphi) \cap L_{3}(\theta)$. In both cases $\omega \in \mathcal{L}_{3}(\varphi * \theta)$ by Table 2 , thus $\omega(\neg(\varphi * \theta))=1$.

If $\omega(\neg(\varphi * \theta))=1$ then $\omega \in L_{3}(\varphi * \theta)$. The only options given in Table 2 are $\omega \in$ $L_{2}(\varphi) \cap L_{3}(\theta)$ and $\omega \in L_{3}(\varphi) \cap L_{3}(\theta)$.

$$
\begin{aligned}
& \omega \in L_{2}(\varphi) \cap L_{3}(\theta) \Longrightarrow \omega\left(\left(\square_{1} \varphi \wedge \square_{1} \neg \varphi\right) \wedge \neg \theta\right)=1 \\
& \omega \in L_{3}(\varphi) \cap L_{3}(\theta) \Longrightarrow \omega(\neg \varphi \wedge \neg \theta)=1
\end{aligned}
$$

hence

$$
\omega\left(\left(\left(\square_{1} \varphi \wedge \square_{1} \neg \varphi\right) \wedge \neg \theta\right) \vee(\neg \varphi \wedge \neg \theta)\right)=1
$$


Lemma 2. If $\star$ is any binary operator on $\mathcal{F}$ satisfying CI1' and CI2' then $\star$ is the Cautious Improvement operator.

Proof: Suppose $\star$ satisfies

$$
\begin{gathered}
(\varphi \wedge \theta) \vee\left(\left(\square_{1} \varphi \wedge \square_{1} \neg \varphi\right) \wedge \theta\right)=\equiv \varphi \star \theta \\
\left(\left(\square_{1} \varphi \wedge \square_{1} \neg \varphi\right) \wedge \neg \theta\right) \vee(\neg \varphi \wedge \neg \theta)=\neg(\varphi \star \theta)
\end{gathered}
$$

We have to prove that $L_{j}(\varphi \star \theta)=L_{j}(\varphi * \theta)$ for $j=1,2,3$, i.e., that $\varphi \star \theta \equiv \varphi * \theta$.

Given any interpretation $\omega$

$$
\begin{aligned}
\omega \in L_{1}(\varphi \star \theta) & \Longleftrightarrow \omega\left((\varphi \wedge \theta) \vee\left(\left(\square_{1} \varphi \wedge \square_{1} \neg \varphi\right) \wedge \theta\right)\right)=1 \\
& \Longleftrightarrow \omega \in L_{1}(\varphi * \theta) \quad(\text { Lemma } 1)
\end{aligned}
$$

similarly,

$$
\begin{aligned}
\omega \in L_{3}(\varphi \star \theta) & \left.\Longleftrightarrow \omega\left(\left(\square_{1} \varphi \wedge \square_{1} \neg \varphi\right) \wedge \neg \theta\right) \vee(\neg \varphi \wedge \neg \theta)\right)=1 \\
& \Longleftrightarrow \omega \in L_{3}(\varphi * \theta) \quad(\text { Lemma } 1)
\end{aligned}
$$

Finally, suppose $\omega \in L_{2}(\varphi \star \theta)$. As we are assuming that $\star$ satisfies CI1' and CI2', this is equivalent to

$$
\begin{array}{r}
\omega\left((\varphi \wedge \theta) \vee\left(\left(\square_{1} \varphi \wedge \square_{1} \neg \varphi\right) \wedge \theta\right)\right) \leq 1 / 2 \\
\text { and } \\
\omega\left(\left(\left(\square_{1} \varphi \wedge \square_{1} \neg \varphi\right) \wedge \neg \theta\right) \vee(\neg \varphi \wedge \neg \theta)\right) \leq 1 / 2
\end{array}
$$

which is equivalent, via Lemma 1 , to

$$
\begin{gathered}
\omega(\varphi * \theta) \leq 1 / 2 \\
\text { and } \\
\omega(\neg(\varphi * \theta)) \leq 1 / 2
\end{gathered}
$$

This happens if, and only if

$$
\begin{gathered}
\omega(\varphi * \theta) \leq 1 / 2 \\
\text { and } \\
\omega(\varphi * \theta) \geq 1 / 2
\end{gathered}
$$

i.e.,

$$
1 / 2 \leq \omega(\varphi * \theta) \leq 1 / 2
$$

or, equivalently, $\omega(\varphi * \theta)=1 / 2$ which is equivalent to $\omega \in L_{2}(\varphi * \theta)$.

Hence $\omega \in L_{2}(\varphi \star \theta)$ iff $\omega \in L_{2}(\varphi * \theta)$ thus $L_{2}(\varphi \star \theta)=L_{2}(\varphi * \theta)$.

Henceforth, $\varphi \star \theta \equiv \varphi * \theta$.

Proof of Theorem 6: It is a consequence of the following two lemmas: 
Lemma 3. The operator $\otimes_{k}$ defined by Table 3 satisfies Postulates $\otimes_{k} 1, \otimes_{k} 2$ and $\otimes_{k} 3$.

Proof: Let us denote:

$$
\begin{aligned}
& \phi_{1}=\varphi \otimes_{k} \theta \\
& \phi_{2}=\square_{1}\left(\varphi \otimes_{k} \theta\right) \wedge \square_{1} \neg\left(\varphi \otimes_{k} \theta\right) \\
& \phi_{3}=\neg\left(\varphi \otimes_{k} \theta\right)
\end{aligned}
$$

Notice that for every interpretation $\omega$ and every $l \in\{1,2,3\}$

$$
\omega\left(\phi_{l}\right)=1 \Longleftrightarrow \omega \in L_{l}\left(\varphi \otimes_{k} \theta\right)
$$

Fix $l \in\{1,2,3\}$. Suppose $\omega$ is an interpretation that satisfies $\bigvee_{1 \leq i \leq j \leq 3} \zeta_{i j}^{l}$. Thus, there is a pair $i, j$ such that $k(i, j)=l$ and $\zeta_{i j}^{l} \neq \perp$, i.e., $\zeta_{i j}^{l}$ is a conjunction $\alpha_{i}^{l} \wedge \beta_{j}^{l}$ such that

$$
\omega\left(\alpha_{i}^{l} \wedge \beta_{j}^{l}\right)=1
$$

That is, $\omega\left(\alpha_{i}^{l}\right)=\omega\left(\beta_{j}^{l}\right)=1$, hence $\omega \in L_{i}(\varphi) \cap L_{j}(\theta)$ and thus $\omega \in L_{k(i, j)}\left(\varphi \otimes_{k} \theta\right)$ by Table 3 and $\omega\left(\phi_{l}\right)=1$.

Conversely, suppose that $\omega\left(\phi_{l}\right)=1$, then $\omega \in L_{l}\left(\varphi \otimes_{k} \theta\right)$. According to the definition of $\otimes_{k}$ by Table 3 there is a pair $i, j$ such that $l=k(i, j)$ thus $\omega \in L_{i}(\varphi) \cap L_{j}(\theta)$. This implies that $\omega\left(\alpha_{i}^{l} \wedge \beta_{j}^{l}\right)=1$, hence

$$
\omega\left(\bigvee_{1 \leq i \leq j \leq 3} \zeta_{i j}^{l}\right)=1
$$

Lemma 4. If an operator $\star$ satisfies Postulates $\otimes_{k} 1, \otimes_{k} 2$ and $\otimes_{k} 3$ then $\star$ and $\otimes_{k}$ are the same operator.

Proof: Analogous to the proof of Lemma 2.

\section{References}

Alchourrón, C. E., Gärdenfors, P., \& Makinson, D. (1985). On the logic of theory change: Partial meet contraction and revision functions. Journal of Symbolic Logic, 50, 510530.

Benferhat, S., Konieczny, S., Papini, O., \& Pino Pérez, R. (2000). Iterated revision by epistemic states: Axioms, semantics and syntax. In Horn, W. (Ed.), Proceedings of the Fourteenth European Conference on Artificial Intelligence, ECAI'2000, pp. 13-17. IOS Press.

Bergmann, M. (2008). An Introduction to Many-valued and Fuzzy Logic. Cambridge University Press.

Bonanno, G. (2005). A simple modal logic for belief revision. Synthese, 147(2), 193-228. 
Bonanno, G. (2007). Axiomatic characterization of the AGM theory of belief revision in a temporal logic. Artif. Intell., 171(2-3), 144-160.

Booth, R., \& Meyer, T. (2006). Admissible and restrained revision. Journal of Artificial Intelligence Research, 26, 127-151.

Booth, R., Meyer, T., \& Wong, K.-S. (2006). A bad day surfing is better than a good day working: How to revise a total preorder. In Doherty, P., Mylopoulos, J., \& Welty, C. A. (Eds.), KR, pp. 230-238. AAAI Press.

Boutilier, C. (1996). Iterated revision and minimal change of conditional beliefs. Journal of Philosophical Logic, 25(3), 262-305.

Darwiche, A., \& Pearl, J. (1997). On the logic of iterated belief revision. Artificial Intelligence, 89, 1-29.

Freund, M., \& Lehmann, D. (2002). Belief revision and rational inference. arXiv:cs0204032v1, 1-25.

Gärdenfors, P. (1988). Knowledge in flux. MIT Press.

Gärdenfors, P. (1991). Belief revision and nonmonotonic logic: Two sides of the same coin?. In van Eick J. (Ed.) Logics in AI. JELIA 1990. Lecture Notes in Computer Science, vol 478 ., pp. 52-54.

Gärdenfors, P. (1992). Belief Revision. Cambridge University Press.

Jin, Y., \& Thielscher, M. (2007). Iterated belief revision, revised. Artificial Intelligence, 171, $1-18$.

Katsuno, H., \& Mendelzon, A. O. (1991). Propositional knowledge base revision and minimal change. Artificial Intelligence, 52, 263-294.

Kleene, S. C. (1938). On notation for ordinal numbers. The Journal of Symbolic Logic, 3, $150-155$.

Konieczny, S., Medina Grespan, M., \& Pino Pérez, R. (2010). Taxonomy of improvement operators and the problem of minimal change. In Proceedings of the Twelfth International Conference on Principles of Knowledge Representation And Reasoning (KR 2010), pp. 161-170.

Konieczny, S., \& Pino Pérez, R. (2008). Improvement operators. In Proceedings of the Eleventh International Conference on Principles of Knowledge Representation And Reasoning (KR 2008), pp. 177-187.

Makinson, D., \& Gärdenfors, P. (1991). Relation between the logic of theory change and nonmonotonic logic. In A. Fuhrmann, M. Morreau (eds.) The Logic of Theory Change: Workshop, Konstanz, FRG, October 13-15, 1989 Proceedings. Lecture Notes in Artificial Intelligence, Volume 465, pp. 185-205.

Medina Grespan, M., \& Pino Pérez, R. (2013). Representation of basic improvement operators. In Trends in Belief Revision and Argumentation Dynamics, pp. 195-227. College Publications.

Nayak, A. C. (1994). Iterated belief change based on epistemic entrenchment. Erkenntnis, 41, 353-390. 
Peppas, P. (2008). Belief revision. In van Harmelen, F., Lifschitz, V., \& Porter, B. W. (Eds.), Handbook of Knowledge Representation, Vol. 3 of Foundations of Artificial Intelligence, pp. 317-359. Elsevier.

Pino Pérez, R., \& Uzcátegui, C. (2010). Dinámica del Conocimiento. Ediciones IVIC.

Rott, H. (2009). Shifting priorities: Simple representations for twenty-seven iterated theory change operators. In Makinson, D., Malinowski, J., \& Wansing, H. (Eds.), Towards Mathematical Philosophy, Vol. 28 of Trends in logic, pp. 269-296. Springer. 